\title{
Supraglottic airway devices for administration of surfactant to newborn infants with respiratory distress syndrome: a narrative review
}

\author{
Calum T Roberts (D) , 1,2,3 Brett James Manley, ${ }^{4,5,6}$ Joyce E O'Shea, ${ }^{7}$ Michael Stark, ${ }^{8,9}$ \\ Chad Andersen (D) , ${ }^{8,9}$ Peter G Davis (D) , ${ }^{4}$ Adam Buckmaster ${ }^{10,11}$
}

For numbered affiliations see end of article.

\section{Correspondence to}

Dr Calum T Roberts, Dept. of Paediatrics, Monash University Faculty of Medicine Nursing and Health Sciences, Clayton, VIC 3168, Australia;

calum.roberts@monash.edu

Received 29 May 2020 Revised 1 September 2020

Accepted 2 September 2020

Published Online First

28 September 2020

\section{Check for updates}

(C) Author(s) (or their employer(s)) 2021. No commercial re-use. See rights and permissions. Published by BMJ.

To cite: Roberts $\mathrm{CT}$, Manley BJ, O'Shea JE, et al. Arch Dis Child Fetal Neonatal Ed 2021;106:F336-F341.

\section{ABSTRACT}

Surfactant is an effective treatment for respiratory distress syndrome, being particularly important for infants in whom continuous positive airway pressure (CPAP) provides insufficient support. Supraglottic airway devices present an attractive option for surfactant delivery, particularly as an alternative to methods dependent on direct laryngoscopy, a procedural skill that is both difficult to learn and in which to maintain competence. Published studies provide encouraging data that surfactant administration by supraglottic airway device can be performed with a high rate of success and may reduce the need for subsequent intubation compared with either continued CPAP or surfactant administration via endotracheal tube. However, existing randomised controlled trials (RCTs) are heterogeneous in design and include just over 350 infants in total. To date, all RCT evidence has been generated in tertiary units, whereas the greatest potential for benefit from the use of these devices is likely to be in non-tertiary settings. Future research should investigate choice and utility of device in addition to safety and effectiveness of procedure. Importantly, studies conducted in non-tertiary settings should evaluate feasibility, meaningful clinical outcomes and the impact that this approach might have on infants and their families. Supraglottic airway devices may represent a simple and effective mode of surfactant administration that can be widely used by a variety of clinicians. However, further well-designed RCTs are required to determine their role, safety and effectiveness in both tertiary and non-tertiary settings before introduction into routine clinical practice.

\section{SURFACTANT TREATMENT FOR RESPIRATORY DISTRESS SYNDROME (RDS)}

Current international recommendations for the treatment of preterm infants with RDS emphasise the use of non-invasive respiratory support, with the aim of avoiding mechanical ventilation (MV) via endotracheal tube. ${ }^{12}$ Approaches favouring non-invasive modalities, specifically nasal continuous positive airway pressure (CPAP), rather than elective intubation, may result in reduced rates of adverse outcomes including death or bronchopulmonary dysplasia. ${ }^{3}$

However, CPAP will provide insufficient support for many infants, resulting in subsequent need for MV. Exposure to MV, and RDS, is associated with increased risk of morbidity and mortality, with particularly high risks seen in low-income and middle-income settings, where mortality can exceed $50 \% .^{4}$

Exogenous surfactant is an effective treatment for RDS, traditionally administered via endotracheal tube after intubation. Approaches combining the benefit of surfactant treatment with that of early CPAP, minimising the likelihood of $\mathrm{MV}$, may reduce the risk of adverse outcomes. These include intubation followed by early extubation (commonly referred to as intubation-surfactant-extubation $(\text { INSURE) })^{5}$ or surfactant administration by a thin catheter during ongoing CPAP support (less invasive surfactant administration (LISA)). ${ }^{67}$ Current European RDS guidelines recommend LISA as 'the preferred mode of surfactant administration for spontaneously breathing babies on CPAP, provided that clinicians are experienced with this technique'. ${ }^{2}$ While these guidelines are prepared for infants of all gestational ages, most LISA trials have included very preterm infants (born $<32$ weeks' gestation), and there are few data relating to moderate to late preterm infants. ${ }^{8}$

Importantly, LISA and INSURE require skill in direct laryngoscopy and are therefore likely to be effective only when performed by operators with substantial experience in this technique (typically from previous endotracheal intubations). Historically, intubation was performed frequently, both at birth, and in tertiary neonatal intensive care units (NICUs) and could be learnt early during paediatric training. However, advances in perinatal care, cessation of routine direct laryngoscopy for infants born through meconium stained liquor and increased reliance on CPAP have resulted in a reduction in both intubation attempts, and success rates, among trainees. A recent study reported success rates of only $49 \%$ on first attempt, $46 \%$ for nonpremedicated intubations in the delivery room and $24 \%$ for the most junior trainees. ${ }^{9}$ Intubation is still commonly performed in tertiary NICUs. However, opportunities to build and maintain competence are much less frequent for clinicians in non-tertiary settings, leading some experts to question whether it remains realistic to expect non-neonatologist paediatricians caring for newborn infants to be proficient in intubation. ${ }^{10} \mathrm{~A}$ study describing the practice of 11 general paediatricians working in an Australian special care nursery (SCN) with $>3500$ births annually found that each individual performed neonatal intubation on average once per year. ${ }^{11}$ 
Adverse events are commonly reported during intubation, complicating $22 \%$ of encounters in one single-centre NICU report. ${ }^{12}$ Success rates are lower, and adverse events more frequent, during intubation without muscle relaxants. Importantly such medications cannot be used during LISA. Anecdotally, laryngoscopy without premedication is more challenging in infants of greater gestational age and birth weight, the population commonly treated in SCNs.

Given that many infants with RDS are cared for in non-tertiary SCNs, identifying a surfactant administration technique best suited to this setting presents an important challenge. In countries such as Australia, neonatal transfers may take place over hundreds of kilometres and may result in separation of infant and mother, or separation of the mother-infant pair from their local support network. If an easier, effective and safe technique for surfactant administration was available in SCNs, this might prevent the need for endotracheal intubation and MV, and subsequent NICU transfer, in some cases. Surfactant nebulisation is an attractive and truly 'non-invasive' option, but suitable devices are not yet widely available. ${ }^{13}$ Supraglottic airway devices incorporate an airway tube attached to a small mask and are inserted without laryngoscopy. ${ }^{14}$ In this review, we will summarise the current evidence for supraglottic airway device use in neonates, specifically as a route for surfactant administration in infants with RDS.

\section{SUPRAGLOTTIC AIRWAY DEVICE USE IN NEONATES}

The laryngeal mask airway (LMA), the first supraglottic airway device, was introduced in the 1980s, with reports of use in infants in the early 1990s. ${ }^{15}$ Initial studies focused on their use as an alternative to intubation or face mask to deliver intermittent positive pressure ventilation (IPPV) during neonatal resuscitation.

A recent Cochrane Review included six randomised controlled trials (RCTs) and one quasirandomised trial comparing IPPV provided by supraglottic airway with use of face mask or intubation. ${ }^{14}$ On pooled analysis of five studies enrolling 661 neonates, the authors found that, compared with face mask, use of supraglottic airway for IPPV was associated with reduced need for endotracheal intubation with risk ratio (RR) 0.24 (95\% CI 0.12 to 0.47$)$ and typical risk difference $-0.10(95 \% \mathrm{CI}-0.14$ to 0.06 ), with number needed to treat 10 . Supraglottic airways were found in two RCTs (108 infants) to offer comparable efficacy to endotracheal intubation. ${ }^{16}{ }^{17}$ These trials reported 90\% first attempt intubation success, considerably higher than rates seen in more recently published data. First attempt success of supraglottic airway insertion, by resuscitators including anaesthetists, nurses and paediatricians was achieved in 287/301 infants $(95 \%) \cdot{ }^{18-20}$ In contrast, studies of neonatal intubation and LISA in tertiary NICUs report first attempt success rates of $44 \%-90 \%{ }^{21-24}$ A meta-analysis of RCTs comparing LISA and intubation for surfactant, including 572 infants, reported firstattempt success rates of $80.6 \%$ and $80.9 \%$, respectively. ${ }^{8}$

The Cochrane Review authors concluded that supraglottic airway use was more effective than face mask for providing IPPV during resuscitation, and of comparable efficacy to endotracheal intubation, but classed the evidence as very low to moderate quality. They recommended more proactive use of supraglottic airway in infants not responding to face mask IPPV or when attempts at endotracheal intubation are unsuccessful but cautioned that there was limited evidence for infants with birth weight $<1500 \mathrm{~g}$ or born $<34$ weeks' gestation. Current international consensus guidance recommends use of supraglottic airway devices during resuscitation for infants born $>34$ weeks' gestation. ${ }^{25}$

\section{CASE REPORTS OF SURFACTANT ADMINISTRATION BY SUPRAGLOTTIC AIRWAY}

The use of a supraglottic airway to administer surfactant was first described in $1992 .{ }^{26}$ In 2004, Brimacombe $e t ~ a l^{27}$ reported this approach in two infants with RDS, noting improvements in oxygen requirement and blood gases, with neither infant receiving MV. Several further case reports and series have been published since, using various supraglottic airway devices (table 1). Included infants ranged from 28 to 40 weeks' gestation and 880-3624 g at birth, with the majority avoiding MV after surfactant treatment, although just 18 infants were included in five publications. ${ }^{27-31}$

\section{RANDOMISED TRIALS OF SURFACTANT ADMINISTRATION BY SUPRAGLOTTIC AIRWAY}

The most recent Cochrane Review of surfactant administration via supraglottic airway, published in 2011, included only one small RCT. ${ }^{32}$ This study of 26 infants randomised infants of $>1200 \mathrm{~g}$ at birth, receiving CPAP with fractional inspired oxygen $\left(\mathrm{FiO}_{2}\right) \quad 0.30-0.60$, to either supraglottic airway surfactant or continued CPAP treatment, with the primary outcome of intubation. The trial was ceased early due to slow recruitment; intubation rates did not differ significantly between intervention and control groups ( $8 \%$ vs $23 \%, \mathrm{p}=0.59)$.

Since 2011, several further RCTs have been published (table 2). The largest of these compared surfactant administration via supraglottic airway versus continued CPAP treatment, in preterm infants $\geq 1250 \mathrm{~g}$ receiving CPAP for $\mathrm{RDS}$ with $\mathrm{FiO}_{2}$ $0.30-0.40 .{ }^{33}$ This study was also ceased early, after recruitment of 103 of the planned 144 infants. Intubation and MV in the first 7 days were significantly reduced in the supraglottic airway surfactant group ( $38 \%$ vs $64 \%, \mathrm{p}<0.01)$. No adverse events were recorded in either group.

Four RCTs, including 228 infants, have compared surfactant administration via supraglottic airway versus administration via

Table 1 Published case reports and series describing surfactant administration by supraglottic airway

\begin{tabular}{|c|c|c|c|c|c|c|}
\hline & $\begin{array}{l}\text { Number of } \\
\text { infants }\end{array}$ & Gestation at birth (weeks) & Birth weight (g) & Device* & Surfactant dose (mg/kg) & MV \\
\hline Brimacombe et $a l^{27}$ & 2 & 30 and 37 & 1360 and 3200 & Classic LMA, size 1 & 75-100 (first dose), 50 (second dose) & $0 / 2$ \\
\hline Trevisanuto et a $\left.\right|^{30}$ & 8 & Range 28-35 & Range $880-2520$ & Classic LMA, size 1 & 100 & $2 / 8$ \\
\hline Micaglio et $a l^{29}$ & 3 & 32,34 and 37 & 1530,2050 and 3500 & ProSeal LMA, size 1 & 100 & Not reported \\
\hline Barbosa et $a^{28}$ & 1 & 31 & 1335 & ProSeal LMA, size 1 & 200 & $0 / 1$ \\
\hline Vannozzi et $\left.a\right|^{31}$ & 4 & Range $34-40$ & Range 1900-3624 & Air-Q LM, size $0.5-1$ & $150-200$ & Not reported \\
\hline
\end{tabular}

*Manufacturer details: Classic LMA and ProSeal LMA, Teleflex Medical, Wayne, Pennsylvania, USA; Air-Q LM, Cookgas LLC, Mercury Medical, Clearwater, Florida, USA.

LMA, laryngeal mask airway; MV, mechanical ventilation. 


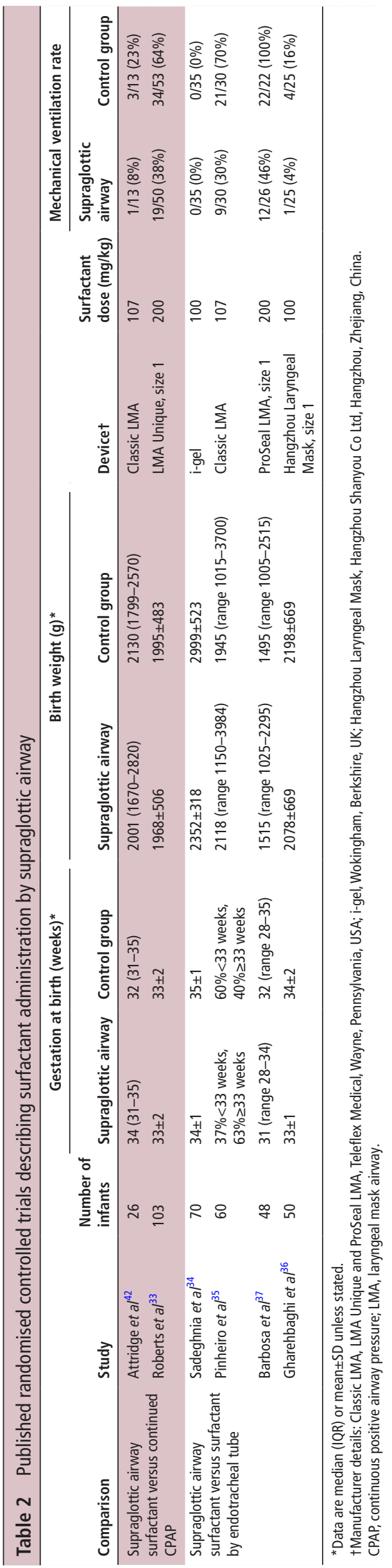

endotracheal tube. In three of these studies, infants randomised to the control group received INSURE. Sadeghnia $e \mathrm{al}^{34}$ studied 70 infants of $\geq 2 \mathrm{~kg}$ at birth, receiving CPAP with $\mathrm{FiO}_{2} \geq 0.30$ for RDS. None of the infants in either group required MV, and other clinical outcomes were similar. Pinheiro et al ${ }^{35}$ included 60 preterm infants $\geq 29$ weeks' gestation with RDS; the supraglottic airway group had a lower rate of intubation $(30 \%$ vs $70 \%, \mathrm{p}<0.01)$. Secondary outcomes and adverse effects did not differ between groups. Gharehbaghi $e t a l^{36}$ reported on 50 infants 33-37 weeks' gestation, and birth weight $\geq 1800 \mathrm{~g}$, treated with surfactant for RDS. Clinical outcomes were similar in the two groups, with $4 \%$ of supraglottic airway infants and $16 \%$ of INSURE infants receiving MV after surfactant $(\mathrm{p}=0.16)$. One RCT compared the use of supraglottic airway surfactant with intubation and continued MV in 48 infants of 28-35 weeks' gestation and $\geq 1000 \mathrm{~g}$ at birth. ${ }^{37}$ Of the 26 infants in the supraglottic airway group, $46 \%$ received MV, while all control group infants received MV as per the trial protocol. Other clinical outcomes were similar.

A recent systematic review and meta-analysis ${ }^{38}$ included five of the RCTs and found supraglottic airway surfactant administration to be associated with a reduction in MV in comparison with both continued CPAP (RR $0.57,95 \%$ CI 0.38 to 0.85 ) and with surfactant administration via endotracheal tube (RR $0.43,95 \%$ CI 0.31 to 0.61 ). However, the authors cautioned that these findings were based on a limited number of infants in studies of varying design, in some cases ceased prior to completion of enrolment, and classed the evidence as very low quality. They recommended that use of surfactant administration via supraglottic airway be limited to clinical trials.

\section{PROCEDURE AND DEVICE \\ Device}

A variety of supraglottic airway devices are available for use in newborn infants and have been included in reports of surfactant administration (tables 1 and 2). The majority of studies exclusively used size one devices (typically the smallest available), although the Air-Q LM is provided in size 0.5 and was used for a single infant in one report. ${ }^{31}$ Size of device is an important issue, given that the incidence and severity of RDS increases with decreasing gestational age and weight. Current evidence comes almost entirely from infants weighing $>1 \mathrm{~kg}$, and it is unclear if available devices could be used successfully in smaller infants. Given the importance of surfactant treatment in the extremely low birthweight population, it is likely that smaller supraglottic airway devices will be needed if their use for this purpose is to become truly widespread.

There are no published studies directly comparing these devices for surfactant administration. However, a manikin study assessing delivery of IPPV using seven different supraglottic airway devices found the i-gel to result in the most effective pressure transmission and lowest measured leak (5.7\%). ${ }^{39}$ Low leak could be beneficial in ensuring effective administration of surfactant from the supraglottic device into the trachea. Two device comparisons conducted in infants receiving IPPV during anaesthesia demonstrated few differences in performance, although the i-gel was associated with faster insertion time than the ProSeal LMA and rated as easier to insert than the Classic LMA. ${ }^{4041}$ Use of an i-gel device to administer surfactant is shown in figure 1.

\section{Premedication and comfort}

With the exception of a single case report of intravenous midazolam use,${ }^{27}$ no studies have reported the use of sedative 


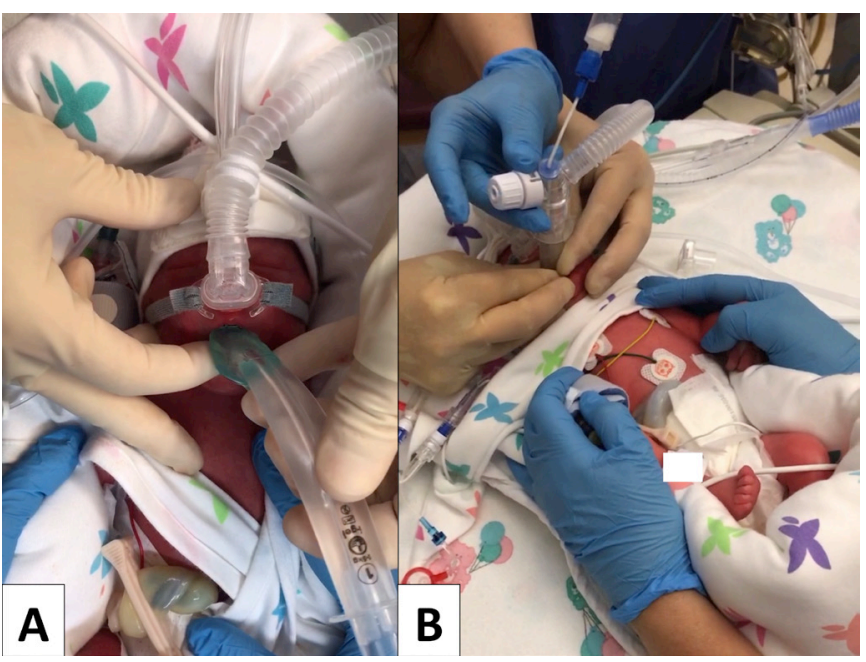

Figure 1 (A) Insertion of i-gel supraglottic airway device in a preterm infant receiving CPAP. (B) Surfactant administration by a flexible catheter inserted into the supraglottic airway device. The catheter length has been measured to reach the distal end of the device lumen. CPAP, continuous positive airway pressure.

medications or muscle relaxants. Comfort measures applied during the procedure include administration of oral sucrose ${ }^{33}$ and application of lignocaine gel to the supraglottic airway device. ${ }^{28} 2937$ Atropine was given, with the aim of reducing the risk of bradycardia in two studies. ${ }^{33} 35$

While many studies anecdotally stated that infants tolerated the procedure well, only one study formally reported pain scoring. The investigators assessed Neonatal Infant Pain Scale immediately before and after surfactant administration, finding similar preprocedure and postprocedure scores in the supraglottic airway group. ${ }^{37}$

\section{Confirmation of position}

When correctly positioned, the mask of the supraglottic airway device sits within the hypopharynx over the laryngeal opening, occluding the oesophagus with a low-pressure seal. ${ }^{19} 20$ Several studies stated that they confirmed correct positioning of the device within the airway with the use of carbon dioxide detection, either using a colorimetric device ${ }^{333536}$ or placement of endtidal monitoring at the end of the surfactant catheter. ${ }^{31}$ Other reported methods included auscultation of breath sounds, ${ }^{36} 42$ observation of chest movement during IPPV ${ }^{35} 36$ and observation of volume changes to a flow-inflating bag during spontaneous breathing. $^{42}$

\section{Respiratory support during procedure}

The majority of studies report using IPPV during and/or immediately after surfactant administration with the aim of promoting distribution of surfactant into the trachea. Although in some cases a Y-piece was used to allow simultaneous surfactant administration and IPPV, the use of positive end expiratory pressure during IPPV was not reported. However, in one series of four infants, surfactant administration was provided to spontaneously breathing infants during administration of CPAP support via the supraglottic airway, without the use of IPPV, with apparent success. ${ }^{31}$

Administration of surfactant during spontaneous breathing has been suggested as a preferable approach to IPPV, as it may reduce volutrauma. Animal and human studies of surfactant administration into the pharynx or by LISA suggest improved surfactant distribution and lung aeration with this approach. ${ }^{43}$ One animal study, conducted in adult rabbits following lung lavage, compared supraglottic airway surfactant administration either with IPPV or via a catheter passed into the supraglottic airway during spontaneous breathing. No differences in oxygenation or lung histopathology were found between the two groups. ${ }^{45}$ Whether this aspect of administration is truly clinically important requires further evaluation.

\section{Surfactant dosing and delivery}

Doses of between $100 \mathrm{mg} / \mathrm{kg}$ and $200 \mathrm{mg} / \mathrm{kg}$ of surfactant have been used almost universally in published studies, consistent with the doses typically applied via endotracheal tube. Current European RDS guidelines recommend $200 \mathrm{mg} / \mathrm{kg}$ for the first 'early rescue' treatment dose. $^{2}$ Repeat dosing of surfactant via supraglottic airway was permitted in three RCTs, and in some cases these infants avoided intubation, although it is difficult to draw conclusions based on the small number of infants treated. ${ }^{34} 3537$ Rates of repeat dosing in the supraglottic airway and endotracheal surfactant groups did not differ.

Published RCTs have all described the insertion of a catheter into the lumen of the supraglottic airway device to deliver the surfactant. ${ }^{38-4246}$ Variations to this approach include placement of the catheter tip at the midpoint ${ }^{46}$ or distal end ${ }^{3840}$ of the airway lumen or to $1 \mathrm{~cm}$ beyond the lumen, extending into the infant's airway. ${ }^{42}$

Multiple studies have reported performing gastric aspiration postprocedure, with the aim of quantifying how much surfactant was not delivered to the lungs. These findings were variable and often did not correlate with need for intubation. ${ }^{33} 372$ The validity of this method is questionable, as it presumes that all aspirated liquid is surfactant and that all surfactant deposited in the stomach will be aspirated, neither of which is likely to be correct. ${ }^{47}$ Surfactant reflux is observed with other modes of administration including INSURE ${ }^{34}$ and LISA, ${ }^{78}$ with treatment success achieved even if a proportion of the administered dose does not reach the lungs.

\section{Adverse events}

Serious adverse events recorded in published studies include physiological instability during the procedure (prolonged bradycardia and/or desaturation), need for emergent intubation, tension pneumothorax and pulmonary haemorrhage. Several studies report that no serious adverse events occurred. ${ }^{33} 34363742$ Those RCTs reporting pneumothorax decribed rates from $0 \%$ to $20 \%$ in infants managed with a supraglottic airway, which in all cases were similar to the rates seen in control infants. ${ }^{33-37} 42$ Some studies described the occurrence of brief desaturation and/ or bradycardia during supraglottic airway insertion, which were managed with IPPV, without explicitly reporting the incidence. $^{3637}$

\section{Training}

Ease of use is frequently cited as an important advantage of supraglottic airway devices over face mask ventilation or endotracheal intubation. Verification of this claim is critical if supraglottic airway devices are to be more readily adopted into neonatal practice, where they have historically been used less frequently than other settings (eg, anaesthetics). During one NICU-based RCT, providers with minimal prior experience of supraglottic airway use could be trained to achieve first attempt success in $69 \%$ of occasions and success within two attempts in $83 \%$, with 
eventual success in all cases. ${ }^{46}$ Median placement time was 28 s. Studies of supraglottic airway use during resuscitation have demonstrated that inexperienced providers can effectively insert the device after brief training, in both manikins ${ }^{48}$ and infants. ${ }^{49}$ These studies include clinicians of varying experience, including medical staff, nurses, nurse practitioners and midwives.

\section{SUMMARY}

Supraglottic airway devices may be a suitable option for delivery of surfactant, as an alternative to methods that are dependent on direct laryngoscopy, a skill that is increasingly difficult to learn and maintain, even for clinicians in tertiary NICUs. Published studies are encouraging, suggesting that supraglottic airway surfactant administration can be performed with a high rate of procedural success and may reduce the need for subsequent intubation relative to continued CPAP or surfactant administration via INSURE. However, given the heterogeneous design and limited number of infants studied, further clinical evidence is required before this approach can be recommended as routine practice. Beyond assessing efficacy, further research should investigate choice of device, whether to instil surfactant during IPPV or spontaneous breathing, surfactant dose and infant comfort during the procedure.

Furthermore, all RCT evidence to date has been generated in NICUs. The greatest potential for benefit for this technique is likely to be elsewhere: in non-tertiary centres and low-income and middle-income settings. In some areas with the capacity to provide both CPAP and surfactant, this approach has already been adopted into clinical care. Such an initiative was introduced in Azerbaijan in late 2018, supported by the Ministry of Health, and the outcomes of this programme will be of great interest. ${ }^{50}$ Studies conducted outside of NICUs will need to evaluate clinical outcomes and whether supraglottic airway use is truly feasible for clinicians in these centres, how to provide education and training, cost-effectiveness and the impact on infants and their families.

Supraglottic airway devices may represent a simple, effective mode of surfactant administration that can be widely applied by clinicians with varying skillsets. Further well-designed RCTs are required to determine whether such an approach can be applied more widely, in both tertiary and non-tertiary settings.

\author{
Author affiliations \\ ${ }^{1}$ Department of Paediatrics, Monash University Faculty of Medicine Nursing and \\ Health Sciences, Clayton, Victoria, Australia \\ ${ }^{2}$ Monash Newborn, Monash Children's Hospital, Clayton, Victoria, Australia \\ ${ }^{3}$ The Ritchie Centre, Hudson Institute of Medical Research, Clayton, Victoria, Australia \\ ${ }^{4}$ Newborn Research Centre and Neonatal Services, The Royal Women's Hospital, \\ Parkville, Victoria, Australia \\ ${ }^{5}$ Clinical Sciences, Murdoch Children's Research Institute, Parkville, Victoria, Australia \\ ${ }^{6}$ Department of Obstetrics and Gynaecology, The University of Melbourne, Parkville, \\ Victoria, Australia \\ ${ }^{7}$ Department of Paediatrics, Royal Hospital for Sick Children, Glasgow, Scotland, \\ United Kingdom \\ ${ }^{8}$ The Robinson Institute, University of Adelaide, Adelaide, South Australia, Australia \\ ${ }^{9}$ Department of Neonatal Medicine, Women's and Children's Hsopital, Adelaide, \\ South Australia, Australia \\ ${ }^{10}$ Women, Children and Families, Central Coast Local Health District, Gosford, New \\ South Wales, Australia \\ ${ }^{11}$ School of Medicine and Public Health, University of Newcastle, Newcastle, New \\ South Wales, Australia
}

Twitter Michael Stark @MichaelStark4

Contributors CTR wrote the first draft of the manuscript. All authors revised the manuscript content.

Funding The authors have not declared a specific grant for this research from any funding agency in the public, commercial or not-for-profit sectors.
Competing interests None declared.

Patient consent for publication Parental/guardian consent obtained.

Provenance and peer review Not commissioned; externally peer reviewed.

Data availability statement Data sharing not applicable as no datasets were generated and/or analysed for this study. N/A.

\section{ORCID iDs}

Calum T Roberts http://orcid.org/0000-0002-9111-5027

Chad Andersen http://orcid.org/0000-0002-1364-4986

Peter G Davis http://orcid.org/0000-0001-6742-7314

\section{REFERENCES}

1 Cummings JJ, Polin RA, Committee on Fetus and Newborn, American Academy of Pediatrics. Noninvasive respiratory support. Pediatrics 2016;137. doi:10.1542/ peds.2015-3758. [Epub ahead of print: 29 Dec 2015].

2 Sweet DG, Carnielli V, Greisen G, et al. European Consensus Guidelines on the Management of Respiratory Distress Syndrome - 2019 Update. Neonatology 2019;115:432-50.

3 Schmölzer GM, Kumar M, Pichler G, et al. Non-Invasive versus invasive respiratory support in preterm infants at birth: systematic review and meta-analysis. BMJ 2013;347: 55980 .

4 Kumar A, Bhat BV. Epidemiology of respiratory distress of newborns. Indian J Pediatr 1996;63:93-8.

5 Verder H, Robertson B, Greisen G, et al. Surfactant therapy and nasal continuous positive airway pressure for newborns with respiratory distress syndrome. DanishSwedish multicenter study Group. N Engl J Med 1994;331:1051-5.

6 Göpel W, Kribs A, Härtel C, et al. Less invasive surfactant administration is associated with improved pulmonary outcomes in spontaneously breathing preterm infants. Acta Paediatr 2015;104:241-6.

7 Dargaville PA, Aiyappan A, Cornelius A, et al. Preliminary evaluation of a new technique of minimally invasive surfactant therapy. Arch Dis Child Fetal Neonatal Ed 2011;96:F243-8.

8 Aldana-Aguirre JC, Pinto M, Featherstone RM, et al. Less invasive surfactant administration versus intubation for surfactant delivery in preterm infants with respiratory distress syndrome: a systematic review and meta-analysis. Arch Dis Child Fetal Neonatal Ed 2017;102:F17-23.

9 Foglia EE, Ades A, Sawyer T, et al. Neonatal intubation practice and outcomes: an international registry study. Pediatrics 2019;143. doi:10.1542/peds.2018-0902. [Epub ahead of print: 11 Dec 2018].

10 O'Donnell CPF. Intubation difficulty in neonatology: are you experienced? Arch Dis Child Fetal Neonatal Ed 2019;104:F458-60.

11 Hardy A, Fuller DG, Forrester M, et al. Survey of procedural and resuscitation requirements for paediatricians working in a non-tertiary centre: implications for training. J Paediatr Child Health 2019;55:441-5.

12 Foglia EE, Ades A, Napolitano N, et al. Factors associated with adverse events during tracheal intubation in the NICU. Neonatology 2015;108:23-9.

13 Minocchieri S, Berry CA, Pillow JJ, et al. Nebulised surfactant to reduce severity of respiratory distress: a blinded, parallel, randomised controlled trial. Arch Dis Child Fetal Neonatal Ed 2019;104:f313-9.

14 Qureshi MJ, Kumar M. Laryngeal mask airway versus bag-mask ventilation or endotracheal intubation for neonatal resuscitation. Cochrane Database Syst Rev 2018:3:CD003314.

15 Paterson SJ, Byrne PJ, Molesky MG, et al. Neonatal resuscitation using the laryngeal mask airway. Anesthesiology 1994;80:1248-53.

16 Esmail N, Saleh M, Ali A. Laryngeal mask airway versus endotracheal intubation for Apgar score improvement in neonatal resuscitation. Egyptian J Anaesthesiol 2002;18:115-21.

17 Yang C, Zhu X, Lin W, et al. Randomized, controlled trial comparing laryngeal mask versus endotracheal intubation during neonatal resuscitation---a secondary publication. BMC Pediatr 2016;16:17.

18 Singh R, Mohan C, Taxak S. Controlled trial to evaluate the use of LMA for neonatal resuscitation. J Anaesthesio/ Clin Pharmacol 2005;21:303-6.

19 Trevisanuto D, Cavallin F, Nguyen LN, et al. Supreme laryngeal mask airway versus face mask during neonatal resuscitation: a randomized controlled trial. J Pediatr 2015;167:286-91.

20 Zhu X-Y, Lin B-C, Zhang Q-S, et al. A prospective evaluation of the efficacy of the laryngeal mask airway during neonatal resuscitation. Resuscitation 2011;82:1405-9.

21 Bao Y, Zhang G, Wu M, et al. A pilot study of less invasive surfactant administration in very preterm infants in a Chinese tertiary center. BMC Pediatr 2015;15:21.

22 Kribs A, Roll C, Göpel W, et al. Nonintubated surfactant application vs conventional therapy in extremely preterm infants: a randomized clinical trial. JAMA Pediatr 2015;169:723-30.

23 Mohammadizadeh M, Ardestani AG, Sadeghnia AR. Early administration of surfactant via a thin intratracheal catheter in preterm infants with respiratory distress syndrome: feasibility and outcome. J Res Pharm Pract 2015;4:31-6. 
24 O'Shea JE, Thio M, Kamlin CO, et al. Videolaryngoscopy to teach neonatal intubation: a randomized trial. Pediatrics 2015:136:912-9.

25 Perlman JM, Wyllie J, Kattwinkel J, et al. Part 7: neonatal resuscitation: 2015 international consensus on cardiopulmonary resuscitation and emergency cardiovascular care science with treatment recommendations. Circulation 2015; 132:S204-41.

26 Eschen C, Verder H, Albertsen P. Laryngeal mask used as a guideway for brief access to the inter-tracheal space in premature infants. Acta Paediatr 1992:81.

27 Brimacombe J, Gandini D, Keller C. The laryngeal mask airway for administration of surfactant in two neonates with respiratory distress syndrome. Paediatr Anaesth 2004;14:188-90.

28 Barbosa RF, Marcatto JdeO, Silva ACSE, et al. ProSealTM laryngeal mask airway for surfactant administration in the treatment of respiratory distress syndrome in a premature infant. Rev Bras Ter Intensiva 2012;24:207-10.

29 Micaglio M, Zanardo V, Ori C, et al. ProSeal LMA for surfactant administration. Paediatr Anaesth 2008;18:91-2.

30 Trevisanuto D, Grazzina N, Ferrarese $\mathrm{P}$, et al. Laryngeal mask airway used as a delivery conduit for the administration of surfactant to preterm infants with respiratory distress syndrome. Biol Neonate 2005;87:217-20.

31 Vannozzi I, Ciantelli M, Moscuzza F, et al. Catheter and laryngeal mask endotracheal surfactant therapy: the CALMEST approach as a novel mist technique. J Matern Fetal Neonatal Med 2017:30:2375-7.

32 Abdel-Latif ME, Osborn DA, Cochrane Neonatal Group. Laryngeal mask airway surfactant administration for prevention of morbidity and mortality in preterm infants with or at risk of respiratory distress syndrome. Cochrane Database Syst Rev 2011;14

33 Roberts KD, Brown R, Lampland AL, et al. Laryngeal mask airway for surfactant administration in neonates: a randomized, controlled trial. J Pediatr 2018;193:40-6.

34 Sadeghnia A, Tanhaei M, Mohammadizadeh M, et al. A comparison of surfactant administration through i-gel and ET-tube in the treatment of respiratory distress syndrome in newborns weighing more than 2000 Grams. Adv Biomed Res 2014;3:160.

35 Pinheiro JMB, Santana-Rivas Q, Pezzano C. Randomized trial of laryngeal mask airway versus endotracheal intubation for surfactant delivery. J Perinatol 2016;36:196-201.

36 Gharehbaghi M, Moghaddam YJ, Radfar R. Comparing the efficacy of surfactant administration by laryngeal mask airway and endotracheal intubation in neonatal respiratory distress syndrome. Crescent J Medical Biol Sci 2018;5:222-7.
37 Barbosa RF, Simões e Silva AC, Silva YP. A randomized controlled trial of the laryngeal mask airway for surfactant administration in neonates. J Pediatr 2017;93:343-50.

38 Calevo MG, Veronese N, Cavallin F, et al. Supraglottic airway devices for surfactant treatment: systematic review and meta-analysis. J Perinatol 2019:39:173-83.

39 Tracy MB, Priyadarshi A, Goel D, et al. How do different brands of size 1 laryngeal mask airway compare with face mask ventilation in a dedicated laryngeal mask airway teaching manikin? Arch Dis Child Fetal Neonatal Ed 2018;103:F271-6.

40 Kayhan GE, Begec Z, Sanli M, et al. Performance of size 1 I-gel compared with size 1 ProSeal laryngeal mask in anesthetized infants and neonates. ScientificWorldJournal 2015;2015:1-6.

41 Kim M-S, Oh J-T, Min JY, et al. A randomised comparison of the i-gel ${ }^{\mathrm{TM}}$ and the laryngeal mask airway Classic ${ }^{\text {TM }}$ in infants. Anaesthesia 2014;69:362-7.

42 Attridge JT, Stewart C, Stukenborg GJ, et al. Administration of rescue surfactant by laryngeal mask airway: lessons from a pilot trial. Am J Perinatol 2013;30:201-6.

43 Bohlin K, Bouhafs RKL, Jarstrand C, et al. Spontaneous breathing or mechanica ventilation alters lung compliance and tissue association of exogenous surfactant in preterm newborn rabbits. Pediatr Res 2005:57:624-30.

44 van der Burg PS, de Jongh FH, Miedema M, et al. Effect of minimally invasive surfactant therapy on lung volume and ventilation in preterm infants. J Pediatr 2016;170:67-72.

45 Bernardo CA, Mariani GL, Virasoro María de Los Ángeles, et al. Surfactant administration through laryngeal mask airway: a randomized controlled study in rabbits. Am J Perinatol 2018;35:669-75

46 Wanous AA, Wey A, Rudser KD, et al. Feasibility of laryngeal mask airway device placement in neonates. Neonatology 2017;111:222-7.

47 Dargaville PA. Administering surfactant without intubation - what does the laryngeal mask offer us? J Pediatr 2017;93:313-6.

48 Pejovic NJ, Trevisanuto D, Nankunda J, et al. Pilot manikin study showed that a supraglottic airway device improved simulated neonatal ventilation in a low-resource setting. Acta Paediatr 2016;105:1440-3.

49 Pejovic NJ, Trevisanuto D, Lubulwa C, et al. Neonatal resuscitation using a laryngeal mask airway: a randomised trial in Uganda. Arch Dis Child 2018:103:255-60.

50 Nematova RI, Guthrie SO. The introduction of the laryngeal mask airway for surfactant administration in neonates with respiratory distress in Azerbaijan. Eurasian J Clin Sci 2019;2:63-7. 